\title{
Acute Lymphoblastic Leukemia in Remission
}

National Cancer Institute

\section{Source}

National Cancer Institute. Acute Lymphoblastic Leukemia in Remission. NCI Thesaurus.

Code C3586.

Acute lymphoblastic leukemia not growing; responding to treatment. 